\title{
Principle of Maximum Entanglement Entropy and Local Physics of Strongly Correlated Materials
}

\author{
Nicola Lanatà, ${ }^{1, *}$ Hugo U. R. Strand, ${ }^{2,3}$ Yongxin Yao, ${ }^{4}$ and Gabriel Kotliar ${ }^{1}$ \\ ${ }^{1}$ Department of Physics and Astronomy, Rutgers University, Piscataway, New Jersey 08856-8019, USA \\ ${ }^{2}$ Department of Physics, University of Gothenburg, SE-412 96 Gothenburg, Sweden \\ ${ }^{3}$ Department of Physics, University of Fribourg, CH-1700 Fribourg, Switzerland \\ ${ }^{4}$ Ames Laboratory-U.S. DOE and Department of Physics and Astronomy, Iowa State University, Ames, Iowa 50011, USA
}

(Received 28 October 2013; revised manuscript received 15 April 2014; published 16 July 2014)

\begin{abstract}
We argue that, because of quantum entanglement, the local physics of strongly correlated materials at zero temperature is described in a very good approximation by a simple generalized Gibbs distribution, which depends on a relatively small number of local quantum thermodynamical potentials. We demonstrate that our statement is exact in certain limits and present numerical calculations of the iron compounds FeSe and $\mathrm{FeTe}$ and of the elemental cerium by employing the Gutzwiller approximation that strongly support our theory in general.
\end{abstract}

DOI: 10.1103/PhysRevLett.113.036402

Strongly correlated materials display an extremely rich variety of phenomena, such as Mott localization and high$T_{c}$ superconductivity, which do not exist in conventional materials. The key element at the basis of the unconventional physics exhibited by strongly correlated materials is that the Coulomb interaction "localizes" part of the electrons, which retain part of their atomic character, making it impossible to describe these systems within a single-particle picture and opening up the possibility of an entirely different class of phenomena.

A fundamental tool for understanding the physics of strongly correlated materials is the so-called "reduced density matrix," which is obtained from the exact density matrix of the solid by tracing over all degrees of freedom except for those of the correlated local orbitals of interest (e.g., the $d$ electrons of a transition-metal compound). In fact, this object encodes the whole local physics of the corresponding electronic degrees of freedom. For instance, it enables us to define the average populations, the mixedvalence character [1,2], and the entanglement entropy [3,4] of the correlated orbitals, which are fundamental concepts in modern condensed matter theory.

The scope of this work is to understand how the reduced density matrix of the correlated electrons is affected by the quantum environment in a solid at zero temperature. Note that, while this is a fundamental problem of great interest, the answer is definitively nontrivial, as the size of the reduced density matrix grows exponentially with the number of correlated orbitals, and the interaction between the local correlated orbitals and their environment is generally very strong and depends both on the chemical composition and on the arrangement of the atoms within the solid.

Let us reformulate the problem from a general perspective, without confining explicitly the discussion to correlated electron systems. We consider a generic "large"
PACS numbers: 71.27.+a, 03.65.Ud, 05.30.Rt, 74.70.Xa

isolated system $\mathcal{U}$ (the lattice) and represent its Hamiltonian as

$$
\hat{\mathcal{H}}_{\mathcal{U}}=\hat{\mathcal{H}}_{\mathcal{S}}+\hat{\mathcal{H}}_{\mathcal{B}}+\hat{\mathcal{H}}_{\mathcal{S B}}
$$

where $\hat{\mathcal{H}}_{\mathcal{S}}$ is the Hamiltonian of a subsystem $\mathcal{S}$ (a subset of local atomic orbitals), $\hat{\mathcal{H}}_{\mathcal{B}}$ represents the Hamiltonian of its environment $\mathcal{B}$, and $\hat{\mathcal{H}}_{\mathcal{S B}}$ represents the interaction between $\mathcal{S}$ and $\mathcal{B}$. Finally, we assume that $\mathcal{U}$ is in the ground state $\left|\Psi_{\mathcal{U}}^{E_{0}}\right\rangle$ of $\hat{\mathcal{H}}_{\mathcal{U}}$, and we consider the corresponding reduced density matrix

$$
\hat{\rho}_{\mathcal{S}}=\operatorname{Tr}_{\mathcal{B}}\left|\Psi_{\mathcal{U}}^{E_{0}}\right\rangle\left\langle\Psi_{\mathcal{U}}^{E_{0}}\right| .
$$

How does $\hat{\rho}_{\mathcal{S}}$ depend on the coupling between $\mathcal{S}$ and its environment?

In this Letter we argue that, because of quantum entanglement, $\hat{\rho}_{\mathcal{S}}$ exhibits thermodynamical properties pertinent to statistical averages. More precisely, we argue that, due to the property of $\left|\Psi_{\mathcal{U}}^{E_{0}}\right\rangle$ to be quantum entangled, $\hat{\rho}_{\mathcal{S}}$ has, approximately, a simple generalized Gibbs form, which depends only on a few local thermodynamical parameters.

Before exposing our theory it is useful to discuss briefly an important recent related result: the canonical-typicality theorem $[5,6]$. This theorem states that, given a system represented as in Eq. (1) — with a very small hybridization $\hat{\mathcal{H}}_{\mathcal{S B}}$ - the reduced density matrix $\hat{\rho}_{\mathcal{S}}$ of any "typical" $\left|\Psi_{\mathcal{U}}^{E}\right\rangle \in \mathcal{U}_{[E, E+d E]}$, where $\mathcal{U}_{[E, E+d E]}$ is the Hilbert subspace generated by the eigenstates of $\hat{\mathcal{H}}_{\mathcal{U}}$ within the energy window $[E, E+d E]$, is

$$
\hat{\rho}_{\mathcal{S}} \propto e^{-\hat{\mathcal{H}}_{\mathcal{S}} / T_{\mathcal{S}}},
$$

where the temperature $T_{\mathcal{S}}$ is determined by the average energy $E_{\mathcal{S}} \equiv \operatorname{Tr}\left[\hat{\rho}_{S} \hat{\mathcal{H}}_{\mathcal{S}}\right]$. Note that the Gibbs form of $\hat{\rho}_{\mathcal{S}}$ 
arises as an individual property of the typical $\left|\Psi_{\mathcal{U}}^{E}\right\rangle$-a pure state-without calling in cause the construction of an ensemble. The key concept underlying this important theorem is quantum entanglement. A simple way to make this interpretation clear is that Eq. (3) is characterized by the condition

$$
S\left[\hat{\rho}_{\mathcal{S}}\right]=\max \left\{S[\hat{\rho}] \mid \hat{\rho} \in \Omega_{\mathcal{S}}, \quad \operatorname{Tr}\left[\hat{\rho} \hat{\mathcal{H}}_{\mathcal{S}}\right]=E_{\mathcal{S}}\right\},
$$

where $S[\hat{\rho}]=-\operatorname{Tr}[\hat{\rho} \log \hat{\rho}]$ is the entanglement entropy of $\mathcal{S}$ and $\Omega_{\mathcal{S}}$ is the set of all of the local (in $\mathcal{S}$ ) density matrices. Since the entanglement entropy is a measure of the quantum entanglement between $\mathcal{S}$ and $\mathcal{B}$, this characterization of $\hat{\rho}_{\mathcal{S}}$ shall be regarded as a consequence of the individual property of the typical $\left|\Psi_{\mathcal{U}}^{E}\right\rangle \in \mathcal{U}_{[E, E+d E]}$ to be highly entangled [7-10].

Let us now drop the assumption that the interaction between $\mathcal{S}$ and $\mathcal{B}$ is small (which is certainly not the case in materials) and focus on our questions concerning the reduced density matrix $\hat{\rho}_{\mathcal{S}}$ of the ground state $\left|\Psi_{\mathcal{U}}^{E_{0}}\right\rangle$ of $\mathcal{U}$; see Eq. (2) and text below. The key message of this work is that, even in this case, a proper generalization of Eq. (3) holds-albeit only approximately.

In order to demonstrate our statement, let us consider the density matrix $\hat{\rho}\left(a_{1}, \ldots, a_{n}\right)$ characterized by the condition

$$
S\left[\hat{\rho}\left(a_{1}, \ldots, a_{n}\right)\right]=\max \left\{S[\hat{\rho}] \mid \operatorname{Tr}\left[\hat{\rho} \hat{A}_{i}\right]=a_{i} \quad \forall i\right\} .
$$

It is known that the solution $\hat{\rho}\left(a_{1}, \ldots, a_{n}\right)$ of Eq. (5) has, if it is nondegenerate, the generalized Gibbs form [11-13]

$$
\hat{\rho}\left(\lambda_{1}, \ldots, \lambda_{n}\right) \propto e^{-\sum_{i=1}^{n} \lambda_{i} \hat{A}_{i}} \equiv e^{-\hat{\mathcal{F}}_{\mathcal{S}}} .
$$

From now on we refer to Eq. (6) as the principle of maximum entanglement entropy (PMEE) relative to the set of observables $\hat{\mathcal{A}}=\left\{\hat{A}_{1}, \ldots, \hat{A}_{n}\right\}$ and to the constraints in Eq. (5) as the corresponding testable information.

A possible way to quantify the goodness of a given PMEE is the following quantity:

$$
\Delta\left[\left\{\hat{A}_{1}, \ldots, \hat{A}_{n}\right\}\right]=\min _{\lambda_{1}, \ldots, \lambda_{n}} D\left[\hat{\rho}\left(\lambda_{1}, \ldots, \lambda_{n}\right), \hat{\rho}_{\mathcal{S}}\right],
$$

where $\hat{\rho}_{\mathcal{S}}$ is the actual reduced density matrix of the system and

$$
D\left[\hat{\rho}_{1}, \hat{\rho}_{2}\right] \equiv \operatorname{Tr}\left(\left|\hat{\rho}_{1}-\hat{\rho}_{2}\right|\right) / 2 \in[0,1]
$$

is a standard trace distance that represents the maximal difference between $\hat{\rho}_{1}$ and $\hat{\rho}_{2}$ in the probability of obtaining any measurement outcome [14].

In summary, we have proposed a systematic method to construct and verify the goodness of a generalized Gibbs ansatz for the reduced density matrix $\hat{\rho}_{\mathcal{S}}$ of a generic system. The key step is the identification of a subset of local observables $\hat{\mathcal{A}} \equiv\left\{\hat{A}_{1}, \ldots, \hat{A}_{n}\right\}$, whose expectation values are expected-e.g., on the basis of physical considerations - to be directly controlled by the system-environment interaction. Note that $\Delta[\hat{\mathcal{A}}] \equiv 0$ in the limit in which $\hat{\mathcal{A}}$ coincides with the set of all of the local observables. In fact, any density matrix is uniquely defined by all of the expectation values of the observables within its Hilbert space.

As we are going to show, the PMEE is a very useful theoretical tool, as a subset $\hat{\mathcal{A}}$ containing only a "few" observables is often sufficient to have $\Delta[\hat{\mathcal{A}}] \simeq 0$. In other words, it is generally possible to define a series of observables $\hat{A}_{i}$ such that the corresponding series of trace distances

$$
\Delta_{n} \equiv \Delta\left[\left\{\hat{A}_{1}, \ldots, \hat{A}_{n}\right\}\right]
$$

converges "rapidly" to zero as a function of $n$, regardless of the details of the environment $\mathcal{B}$ and its coupling with $\mathcal{S}$.

We point out that the PMEE [see Eq. (6)] has a twofold interpretation: (i) the only "relevant" testable information of $\hat{\rho}_{\mathcal{S}}$ consists in the expectation values $a_{i}$ of the observables $\hat{A}_{i} \in \hat{\mathcal{A}}$; (ii) the $\mathcal{S}$ degrees of freedom are essentially in a Gibbs state, but they experience the effective interaction encoded in a "renormalized" local Hamiltonian $\hat{\mathcal{F}}_{\mathcal{S}}$ that is generally different from the original $\hat{\mathcal{H}}_{\mathcal{S}}$. This twofold interpretation reflects the Legendre duality between the expectation values $a_{i}$ and the corresponding generalized chemical potentials $\lambda_{i}$.

Strongly correlated electron systems. - From now on we restrict our attention to many-body correlated electron systems in their ground state. More precisely, we consider a generic multiband Hubbard model (HM)

$$
\hat{\mathcal{H}}_{\mathcal{U}}=\sum_{i \neq j} \sum_{a, b=1}^{\nu} \epsilon_{i j}^{a b} c_{i a}^{\dagger} c_{j b}+\sum_{i} \hat{\mathcal{H}}_{i}^{\text {loc }}\left[\left\{c_{i c}^{\dagger}\right\},\left\{c_{i c}\right\}\right],
$$

where $i$ and $j$ are "site" labels and $a, b, c=1, \ldots, \nu$ label both the spin $\sigma$ and the orbital $m$. The Hamiltonian $\hat{\mathcal{H}}_{\mathcal{U}}$ can be separated as in Eq. (1), with $\hat{\mathcal{H}}_{\mathcal{S}}$ corresponding to the $i$-local operator $\hat{\mathcal{H}}_{i}^{\text {loc }}$ - which, in general, can include both a quadratic term $\hat{\mathcal{H}}_{i}^{\epsilon}$ and a quartic term $\hat{\mathcal{H}}_{i}^{\text {int }}$ (representing the on-site Coulomb interaction).

In order to define a PMEE for the $\mathcal{S}$ reduced density matrix, we need to understand which local observables have to be included in $\hat{\mathcal{A}}$ [see Eq. (6) and text below] to describe approximately the local physics of the system.

Because of the coupling between the environment and the local space, the expectation value of $\hat{\mathcal{H}}_{\mathcal{S}}$ with respect to $\hat{\rho}_{\mathcal{S}}$ is controlled by their reciprocal interaction. This implies that $\hat{\mathcal{H}}_{\mathcal{S}}$ has to be included in $\hat{\mathcal{A}}$. On the other hand, since $\hat{\mathcal{H}}_{\mathcal{S B}}$ is not generally small, there are at least two additional key physical mechanisms that our PMEE shall take into 
account. (I) Because of the hybridization effect, also the individual local orbital populations are controlled by the coupling with the environment. (II) We expect that the effective local interaction $\hat{\mathcal{F}}_{\mathcal{S}}$ experienced by the local degrees of freedom is renormalized. Furthermore, we expect that $\hat{\mathcal{F}}_{\mathcal{S}}$ is not isotropic but is invariant only under the point group of the system.

From the above heuristic arguments, we conclude that $\hat{\mathcal{A}}$ should include at least all of the quadratic and quartic operators compatible with the symmetry of the system. According to our scheme, the corresponding PMEE is $\hat{\rho}_{\mathcal{S}}^{\text {fit }} \propto e^{-\hat{\mathcal{F}}_{\mathcal{S}}}$ [see Eq. (6)], where $\hat{\mathcal{F}}_{\mathcal{S}}$ is the most general linear combination of quadratic and quartic operators. Note that $\hat{\rho}_{\mathcal{S}}^{\text {fit }}$ is an extremely "special" density matrix, as the number of parameters that determine it grows only quartically with $\nu$ [see Eq. (10)] rather than exponentially. Finally, we point out that the PMEE $\hat{\rho}_{\mathcal{S}}^{\text {fit }}$ is exact not only in the so-called "atomic limit" $\hat{\mathcal{H}}_{\mathcal{S B}} \rightarrow 0$, but also for any quadratic $\hat{\mathcal{H}}_{\mathcal{U}}$ [15]. In fact, Wick's theorem ensures that the expectation value of any local observable depends only on the "Wick's contractions" $\left\langle\Psi_{\mathcal{U}}^{E_{0}}\left|c_{\mathcal{S} a}^{\dagger} c_{\mathcal{S} b}\right| \Psi_{\mathcal{U}}^{E_{0}}\right\rangle$, that can be reproduced exactly by a proper quadratic $\hat{\mathcal{F}}_{\mathcal{S}}$.

For later convenience, we define the following series of PMEE for the local reduced density matrix $\hat{\rho}_{\mathcal{S}}$ of a generic HM: (i) $\Delta_{1}$, corresponding to $\hat{\mathcal{A}}_{1} \equiv\left\{\hat{\mathcal{H}}^{\text {loc }}, \hat{N}\right\}$, where $\hat{\mathcal{H}}^{\text {loc }}$ is the on-site Hamiltonian and $\hat{N}$ is the number operator of the $\mathcal{S}$ electrons; (ii) $\Delta_{2}$, corresponding to $\hat{\mathcal{A}}_{2}$ containing $\hat{\mathcal{H}}^{\text {loc }}$ and all of the quadratic operators commuting with the point group of the system; and (iii) $\Delta_{3}$, corresponding to $\hat{\mathcal{A}}_{3}$ containing all of the quadratic and the quartic operators that commute with the point group of the system. As a reference, it is also useful to define the trace distance $\Delta_{0}$ between $\hat{\rho}_{\mathcal{S}}$ and the maximally entangled state, which is the local density matrix proportional to the identity-that corresponds to the PMEE for an empty set of observables, $\hat{\mathcal{A}}_{0} \equiv\{\}$.

Numerical benchmarks.-In order to benchmark our theory and demonstrate its usefulness for the study of materials, here we consider, as a first example, the reduced local density matrix $\hat{\rho}_{d}$ of a realistic HM representing the iron compound FeSe. Additional benchmark calculations of FeTe and of the elemental cerium are discussed in the Supplemental Material [16].

We construct the HM of FeSe by adopting the same band structure $\epsilon$ [see Eq. (10)] used in Ref. [17], which was generated by using density functional theory with the generalized gradient approximation for the exchangecorrelation potential, according to the Perdew-BurkeErnzerhof recipe implemented in QUANTUM ESPRESSO [18] and by applying WANNIER90 [19] to compute the maximally localized Wannier orbitals. Finally, we make use of the Slater parametrization of the on-site interaction $\hat{\mathcal{H}}^{\text {int }}$. Since the HM cannot be solved exactly, we solve it approximately within the Gutzwiller approximation (GA) [20], which is a very reliable approximation for the ground state of correlated metals. In particular, we employ the numerical implementation developed in Refs. [21-24].

In the first panel of Fig. 1, the quasiparticle renormalization weights are shown as a function of the interaction strength $U$, keeping the ratio $J / U$ fixed at 0.224 and $1 / \gamma=0.25$; see Ref. [25]. As discussed in Ref. [17], at $U \simeq 2 \mathrm{eV}$ the system undergoes a clear crossover from a normal metallic phase $(Z \simeq 1)$ toward a bad-metallic phase $(Z \ll 1)$ - the so-called Janus phase [26]. Our purpose is to analyze the local reduced density matrix $\hat{\rho}_{d}$ of the Fe $d$ electrons and to verify the goodness of the PMEE for our FeSe HM, both in the normal-metal regime and in the Janus phase.

In the second panel of Fig. 1 is shown the evolution of the PMEE trace distances $\Delta_{0}, \Delta_{1}, \Delta_{2}$, and $\Delta_{3}$. The corresponding series $\Delta_{n}$ [see Eq. (9)] is shown explicitly in the inset for three values of $U$ as a function of the respective number $\nu_{n}$ of fitting parameters required. Remarkably, $\Delta_{n}$ converges very rapidly to 0 for all $U$ 's; see Eq. (9). In fact, although the number of independent parameters of $\hat{\rho}_{d}$ is 2516 , the $\Delta_{3}$ PMEE, which is defined by only 53 free parameters, is sufficient to obtain a very accurate fit for every $U$ considered-as indicated by the trace distance $\Delta_{3} \ll 1$.

In order to get an even better idea of how accurate our PMEE fits are, we show also the histogram of the local configuration probabilities of the eigenstates of $\hat{\mathcal{H}}^{\text {loc}}$ :

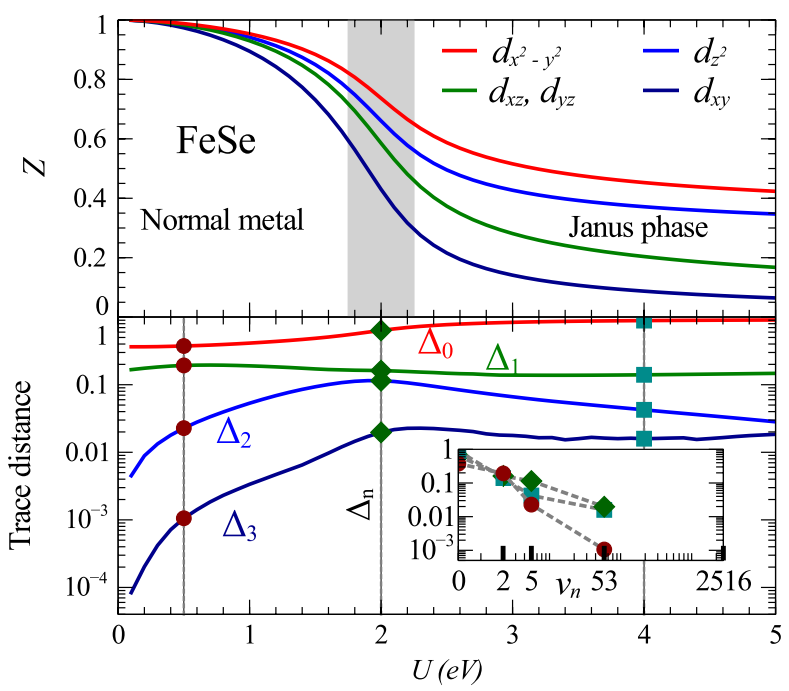

FIG. 1 (color online). Upper panel: quasiparticle renormalization weights of FeSe. The normal-metal phase (small $U$ ) and the Janus phase (large $U$ ) are indicatively separated by a vertical shaded line. Lower panel: PMEE trace distances $\Delta_{n}$ for the reduced density matrix $\rho_{d}$ of FeSe. The series shown in the insets correspond to $U=0.5 \mathrm{eV}, U=2 \mathrm{eV}$, and $U=4 \mathrm{eV}$. All the calculations are performed at fixed $J / U=0.224$. 


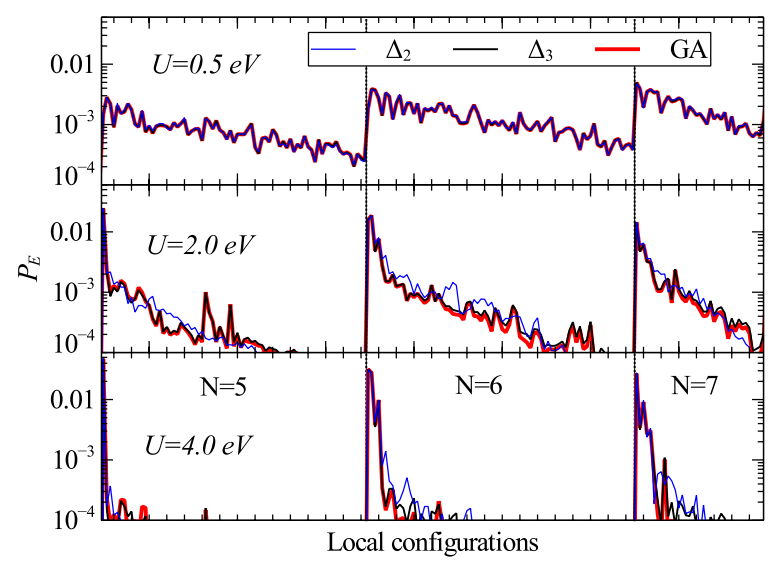

FIG. 2 (color online). Local configuration probabilities $P_{E}$ of the eigenstates of $\hat{\mathcal{H}}^{\text {loc }}$ of FeSe, in the sectors $N=5,6,7$, for $U=0.5 \mathrm{eV}, \quad U=2 \mathrm{eV}$, and $U=4 \mathrm{eV}$ at fixed $J / U=0.224$. The GA configuration probabilities (red line) are shown in comparison with the local configuration probabilities evaluated by using the PMEE density matrices corresponding to the distances $\Delta_{2}$ and $\Delta_{3}$. Within each $N$ sector, the configuration probabilities $P_{E}$ are sorted in ascending order of energy $E \equiv\left\langle\psi_{E}\left|\hat{\mathcal{H}}^{\text {loc }}\right| \psi_{E}\right\rangle$, where $\left|\psi_{E}\right\rangle$ are the eigenstates of $\hat{\mathcal{H}}^{\text {loc }}$.

$$
P_{E} \equiv \operatorname{Tr}\left[\hat{\rho}_{d} \hat{P}_{E}\right] / d_{E}
$$

where $\hat{P}_{E}$ is the orthonormal projector over the $E$ eigenspace of $\hat{\mathcal{H}}^{\text {loc }}$ and $d_{E}$ is its degeneracy. In Fig. 2, the computed GA configuration probabilities are shown for three values of $U$ in comparison with the local configuration probabilities evaluated by using the PMEE density matrices corresponding to the trace distances $\Delta_{2}$ and $\Delta_{3}$. These data confirm the goodness of our PMEE. In fact, the structure of the computed local configuration probabilities, which is extremely complex, is captured in detail by the $\Delta_{3}$ PMEE, and this agreement is verified for all $U$ 's, even though the system undergoes a clear crossover between two electronically distinct phases at $U \simeq 2$.

In the Supplemental Material [16], we discuss also first principles calculations of FeTe and of the elemental cerium, which further support our theory. Furthermore, we show that both (i) the crossover between the normal-metal and the Janus phase in the iron chalcogenides and (ii) the $\gamma-\alpha$ isostructural transition of cerium [4] can be neatly understood in terms of the behavior of the PMEE local thermodynamical parameters.

Note that the concept of a generalized Gibbs ansatz has been previously introduced also in the context of systems out of equilibrium [27-31] and interpreted in terms of a principle of maximum entropy subject to given constraints [32]. On the other hand, it must be noted that what we have proposed here is not a principle of maximum entropy but a principle of maximum entanglement entropy (i.e., the entropy of a given subsystem). We point out that, although the formal definitions of entanglement entropy and entropy are mathematically similar, the behavior and the physical meaning of these two quantities are completely different. For example, as previously pointed out, although the systems considered in this work were assumed to be at zero temperature (and consequently with zero entropy), their entanglement entropy was finite because of the quantum entanglement.

Conclusions.-We have shown that the local physics of strongly correlated materials at zero temperature is described by a simple universal generalized Gibbs distribution. This statement is deeply significant, as the interaction between the subsystem (a given atom) and its environment (all of the other atoms) is definitively nonnegligible in real materials. Our finding provides a very powerful theoretical viewpoint on strongly correlated electron systems. In fact, as shown explicitly by our calculations, the simple exponential form of the reduced density matrix enables us to understand in terms of a few local thermodynamical parameters the behavior of many important physical quantities, such as all of the many-body local configuration probabilities of the correlated electrons-whose number is extremely large, in general, as it grows exponentially with the number of correlated orbitals. Our finding might open up the possibility to engineer compounds with desired physical local properties by directly controlling the local thermodynamical parameters, e.g., through proper structure modifications. Furthermore, it might represent a new paradigm for numerical methods involving the reduced density matrix in the computational procedure (such as the GA). Finally, since our theory is based only on the concept of quantum entanglement, it might be applicable not only to materials science, but also to other fields such as quantum thermodynamics [33] and out-of-equilibrium quantum systems. For instance, the principle of maximum entanglement entropy might constitute a bridge between the concepts of "spreading of entanglement" [34] and "thermalization" in nonintegrable systems.

We thank Sheldon Goldstein, Xiaoyu Deng, Luca de' Medici, Giovanni Morchio, Michele Fabrizio, CaiZhuang Wang, and Kai-Ming Ho for useful discussions. N. L. and G. K. were supported by NSF Grant No. DMR1308141. The collaboration was supported by the U.S. Department of Energy through the Computational Materials and Chemical Sciences Network CMSCN. Research at Ames Laboratory is supported by the U.S. Department of Energy, Office of Basic Energy Sciences, Division of Materials Sciences and Engineering. Ames Laboratory is operated for the U.S. Department of Energy by Iowa State University under Contract No. DE-AC0207CH11358. H. U.R. S. acknowledges the support of the Mathematics-Physics Platform $\left(\mathcal{M P}^{2}\right)$ at the University of Gothenburg. Simulations were performed on resources provided by the Swedish National Infrastructure for Computing (SNIC) at Chalmers Centre for Computational Science and Engineering (C3SE) (Project No. 01-11-297). 
*Corresponding author. lanata@physics.rutgers.edu

[1] C. M. Varma, Rev. Mod. Phys. 48, 219 (1976).

[2] J. H. Shim, K. Haule, and G. Kotliar, Nature (London) 446, 513 (2007).

[3] A. Wehrl, Rev. Mod. Phys. 50, 221 (1978).

[4] N. Lanatà, Y. X. Yao, C. Z. Wang, K. M. Ho, J. Schmalian, K. Haule, and G. Kotliar, Phys. Rev. Lett. 111, 196801 (2013).

[5] S. Goldstein, J. L. Lebowitz, R. Tumulka, and N. Zanghì, Phys. Rev. Lett. 96, 050403 (2006).

[6] S. Popescu, A. J. Short, and A. Winter, Nat. Phys. 2, 754 (2006).

[7] D. Gross, S. T. Flammia, and J. Eisert, Phys. Rev. Lett. 102, 190501 (2009).

[8] C. Nadal, S. N. Majumdar, and M. Vergassola, Phys. Rev. Lett. 104, 110501 (2010).

[9] C. Nadal, S. N. Majumdar, and M. Vergassola, J. Stat. Phys. 142, 403 (2011).

[10] P. Hayden, D. W. Leung, and A. Winter, Commun. Math. Phys. 265, 95 (2006).

[11] E. H. Wichmann, J. Math. Phys. (N.Y.) 4, 884 (1963).

[12] E. Lubkin, J. Math. Phys. (N.Y.) 17, 753 (1976).

[13] E. Lubkin, J. Math. Phys. (N.Y.) 18, 345 (1977).

[14] M. A. Nielsen and I. L. Chuang, Quantum Computation and Quantum Information (Cambridge University Press, Cambridge, England, 2000).

[15] I. Peschel and V. Eisler, J. Phys. A 42, 504003 (2009).

[16] See Supplemental Material at http://link.aps.org/ supplemental/10.1103/PhysRevLett.113.036402 for additional benchmark calculations of FeSe, FeTe, and elemental Ce.
[17] N. Lanatà, H. U. R. Strand, G. Giovannetti, B. Hellsing, L. de' Medici, and M. Capone, Phys. Rev. B 87, 045122 (2013).

[18] P. Giannozzi et al., J. Phys. Condens. Matter 21, 395502 (2009).

[19] A. A. Mostofi, J. R. Yates, Y.-S. Lee, I. Souza, D. Vanderbilt, and N. Marzari, Comput. Phys. Commun. 178, 685 (2008).

[20] M. C. Gutzwiller, Phys. Rev. 137, A1726 (1965).

[21] X. Y. Deng, L. Wang, X. Dai, and Z. Fang, Phys. Rev. B 79, 075114 (2009).

[22] N. Lanatà, H. U. R. Strand, X. Dai, and B. Hellsing, Phys. Rev. B 85, 035133 (2012).

[23] H. U. R. Strand, N. Lanatà, M. Granath, and B. Hellsing (unpublished).

[24] N. Lanatà, Y. X. Yao, and G. Kotliar, arXiv:1405.6934.

[25] H. U. R. Strand, arXiv:1303.4215.

[26] L. de' Medici, J. Mravlje, and A. Georges, Phys. Rev. Lett. 107, 256401 (2011).

[27] A. Polkovnikov, K. Sengupta, A. Silva, and M. Vengalattore, Rev. Mod. Phys. 83, 863 (2011).

[28] P. Calabrese, F. H. L. Essler, and M. Fagotti, Phys. Rev. Lett. 106, 227203 (2011).

[29] M. Rigol, V. Dunjko, and M. Olshanii, Nature (London) 452, 854 (2008).

[30] M. Rigol, V. Dunjko, V. Yurovsky, and M. Olshanii, Phys. Rev. Lett. 98, 050405 (2007).

[31] M. A. Cazalilla, Phys. Rev. Lett. 97, 156403 (2006).

[32] E. T. Jaynes, Phys. Rev. 106, 620 (1957).

[33] J. Oppenheim, M. Horodecki, P. Horodecki, and R. Horodecki, Phys. Rev. Lett. 89, 180402 (2002).

[34] H. Kim and D. A. Huse, Phys. Rev. Lett. 111, 127205 (2013). 